\title{
Correction to: Influence of summer conditions on surface water properties and phytoplankton productivity in embayments of the South Shetland Islands
}

\author{
Claudia Aracena' ${ }^{1}$ (1) Humberto E. González ${ }^{2,3}$. José Garcés-Vargas ${ }^{2,3} \cdot$ Carina B. Lange $^{3,4,5} \cdot$ Silvio Pantoja ${ }^{4,5}$. \\ Francisca Muñoz ${ }^{6} \cdot$ Elisabeth Teca $^{2} \cdot$ Eduardo Tejos $^{7}$
}

Published online: 27 June 2018

(c) Springer-Verlag GmbH Germany, part of Springer Nature 2018

\section{Correction to: Polar Biol https://doi.org/10.1007/s00300-018-2338-x}

This correction serves to provide the correct rendering of Table 3 with its respective symbols corrected to superscript (provided below and not in the original article). Additionally with this correction the author would like to bring to attention the revision of the original article, correcting typographical errors in the Abstract, Tables 1, 2, 3 and Figure 7. For the abstract this entailed the changing of 'productivityon' to simply 'productivity'. For Tables 1, 2 and 3, this entailed the changing of "five" to "four". And for Fig. 7 this entailed changing the footnote from "at the five of the SSI" to "at the five stations of this study". The correct legend information for Table 3 should read as follows:
The original article can be found online at https://doi.org/10.1007/ s00300-018-2338-x.

Claudia Aracena

claudia.aracena@umag.cl

1 Centro de Investigación GAIA Antártica, Universidad de Magallanes, Avenida Bulnes 01855, Casilla 113 D, Punta Arenas, Chile

2 Instituto de Ciencias Marinas y Limnológicas, Universidad Austral de Chile, Campus Isla Teja, Edificio Emilio Pugín, Valdivia, Chile

3 Centro FONDAP de Investigación en Dinámica de Ecosistemas Marinos de Altas Latitudes (IDEAL), Universidad Austral de Chile, Campus Isla Teja, Edificio Emilio Pugín, Valdivia, Chile
Table 3 Values of productivity variables in the surface water column integrated from 1 to $25 \mathrm{~m}$ depths at the four stations in the South Shetland Islands.

Additionally the legend for Figure 7 should read:

Fig. 7 Correlations between a nanoplankton Chl- $a$ size fraction percentage (\%) and salinity, and $\mathbf{b}$ nanoplankton Chl- $a$ size fraction percentage (\%) and turbidity at the five stations of this study. $B F$ Fildes Bay, $B C$ Chile Bay, $B H$ Hanna Bay, $B D$ Deception Bay, $R C$ Rada Covadonga.

The author and the copyeditors apologize for the mistakes in the original version of this article. This correction stands to correct the original article.

4 Centro COPAS Sur-Austral, Universidad de Concepción, Casilla 160-C, Concepción, Chile

5 Departamento de Oceanografía, Universidad de Concepción, Casilla 160-C, Concepción, Chile

6 Plancton Andino SpA, Terraplén \# 869, Puerto Varas, Chile

7 Laboratorio de Estudios Ambientales, Departamento de Química Ambiental, Universidad Católica de la Santísima Concepción, Casilla 297, Concepción, Chile 
Table 3 Values of productivity variables in the surface water column integrated from 1 to $25 \mathrm{~m}$ depths at the four stations in the South Shetland Islands

\begin{tabular}{|c|c|c|c|c|c|c|c|c|c|}
\hline Station & $\begin{array}{l}\text { Chl- } a \text { total } \\
\left(\mathrm{mg} \mathrm{m}^{-2}\right)\end{array}$ & $\begin{array}{l}\text { Chl- } a>20 \mu \mathrm{m} \\
\left(\mathrm{mg} \mathrm{m}^{-2}\right)\end{array}$ & $\begin{array}{l}\text { Chl- } a \text { 20-2 } \mu \mathrm{m} \\
\left(\mathrm{mg} \mathrm{m}^{-2}\right)\end{array}$ & $\begin{array}{l}\text { Chl- } a<2 \mu \mathrm{m} \\
\left(\mathrm{mg} \mathrm{m}^{-2}\right)\end{array}$ & $\begin{array}{l}\text { Micro- } \\
\text { plankton \% }\end{array}$ & $\begin{array}{l}\text { Nano- } \\
\text { plankton \% }\end{array}$ & $\begin{array}{l}\text { Picoplank- } \\
\text { ton \% }\end{array}$ & $\begin{array}{l}\text { Total phyto- } \\
\text { plankton (mg } \\
\left.\mathrm{C} \mathrm{m}^{-2}\right)\end{array}$ & $\begin{array}{l}\text { Centric } \\
\text { diatoms } \\
(\mathrm{mg} \mathrm{C} \\
\left.\mathrm{m}^{-2}\right)\end{array}$ \\
\hline $\mathrm{BF}$ & 74.6 & 21.5 & 49.3 & 3.8 & 28.8 & 66.1 & 5.1 & 154.6 & 141.4 \\
\hline $\mathrm{BC}$ & 28.1 & 3.9 & 22.3 & 1.9 & 13.9 & 79.4 & 6.6 & 23.6 & 19.7 \\
\hline $\mathrm{BH}$ & 26.1 & 10.6 & 13.3 & 2.2 & 40.6 & 50.9 & 8.5 & 16.8 & 13.2 \\
\hline $\mathrm{BD}$ & 117.4 & 8.0 & 104.0 & 5.4 & 6.8 & 88.6 & 4.6 & 474.2 & 472.6 \\
\hline $\mathrm{RC}$ & 7.9 & 3.8 & 2.7 & 1.4 & 48.0 & 34.1 & 17.9 & 13.1 & 12.7 \\
\hline Station & $\begin{array}{l}\text { Pennate } \\
\text { diatoms } \\
\left(\mathrm{mg} \mathrm{C} \mathrm{m}^{-2}\right)\end{array}$ & $\begin{array}{l}\text { Dinoflagellates } \\
\left(\mathrm{mg} \mathrm{C} \mathrm{m}^{-2}\right)\end{array}$ & $\begin{array}{l}\text { Ciliates (mg C } \\
\mathrm{m}^{-2} \text { ) }\end{array}$ & $\begin{array}{l}\text { Total microzoo- } \\
\text { plankton (mg C } \\
\mathrm{m}^{-2} \text { ) }\end{array}$ & $\begin{array}{l}\text { Total bac- } \\
\text { terioplank- } \\
\text { ton }(\mathrm{mg} \mathrm{C} \\
\left.\mathrm{m}^{-2}\right)\end{array}$ & $\begin{array}{l}\text { Total bio- } \\
\text { mass (mg } \\
\left.\mathrm{C} \mathrm{m}^{-2}\right)\end{array}$ & $\begin{array}{l}\text { Total phy- } \\
\text { toplankton } \\
\%\end{array}$ & $\begin{array}{l}\text { Total bac- } \\
\text { terioplank- } \\
\text { ton } \%\end{array}$ & $\begin{array}{l}\text { Total } \\
\text { micro- } \\
\text { zooplank- } \\
\text { ton \% }\end{array}$ \\
\hline $\mathrm{BF}$ & 13.2 & 5.2 & 4.2 & 9.4 & 41.1 & 205.1 & 75.4 & 20.0 & 4.59 \\
\hline $\mathrm{BC}$ & 3.8 & 1.0 & 8.2 & 9.2 & 36.9 & 69.7 & 33.8 & 52.9 & 13.26 \\
\hline $\mathrm{BH}$ & 3.5 & 2.4 & 3.8 & 6.2 & 33.4 & 56.4 & 29.8 & 59.2 & 11.05 \\
\hline $\mathrm{BD}$ & 1.6 & 3.7 & 20.6 & 24.4 & 126.6 & 625.2 & 75.8 & 20.3 & 3.90 \\
\hline $\mathrm{RC}$ & 0.4 & 1.3 & 0.4 & 1.7 & 13.0 & 27.8 & 47.0 & 46.8 & 6.20 \\
\hline
\end{tabular}

$B F$ Fildes Bay, $B C$ Chile Bay, $B H$ Hanna Bay, $B D$ Deception Bay, $R C$ Rada Covadonga. Total and fractionated chlorophyll- $a($ Chl- $a)$; total biomass of the main groups of phytoplankton and microzooplankton, and bacterioplankton 